\author{
A. V. NAGAeV (Toruń) \\ S. A. NAgaev (Wien)
}

\title{
ASYMPTOTICS OF RISKLESS PROFIT UNDER SELLING OF DISCRETE TIME CALL OPTIONS
}

Abstract. A discrete time model of financial market is considered. In the focus of attention is the guaranteed profit of the investor which arises when the jumps of the stock price are bounded. The limit distribution of the profit as the model becomes closer to the classic model of geometrical Brownian motion is established. It is of interest that the approximating continuous time model does not assume any such profit.

1. Introduction. Consider the simplest financial market in which securities of two types are circulating. The price evolution of the securities of the first type is given by the equations

$$
b_{k}=b_{0} \varrho^{k}, \quad k=0,1,2, \ldots,
$$

where $b_{0}>0, \varrho>1$. The prices are registered at the equidistant times $t_{k}=a+k h$. With no loss of generality we put $a=0, h=1$, i.e. $t_{k}=k$.

The price of the security of the second type at time $k$ is represented as

$$
s_{k}=s_{0} \xi_{1} \ldots \xi_{k}, \quad k=0,1,2, \ldots,
$$

where the relative jumps $\xi_{k}$ are random.

The securities of the first type are riskless having the interest rate $(\varrho-1) \cdot 100 \%$. Let us call them conventionally bonds. It is clear that possessing securities of the second type is concerned with a risk of their devaluation. We call them stocks.

Taken together in certain amounts $\beta$ and $\gamma$, the securities of both types constitute the so-called portfolio (writer's investment portfolio) whose worth at time $k$ is $\beta b_{k}+\gamma s_{k}$. Playing in the financial market considered consists in successive changes of the portfolio at $k=1, \ldots, n-1$. The successive

2000 Mathematics Subject Classification: Primary 62P05; Secondary 60F17, 60G50.

Key words and phrases: asymptotic uniformity, weak convergence in $D[0,1]$. 
pairs $\left(\beta_{0}, \gamma_{0}\right),\left(\beta_{1}, \gamma_{1}\right), \ldots,\left(\beta_{n-1}, \gamma_{n-1}\right)$ constitute the so-called strategy of the game. Obviously, the basis for choosing $\left(\beta_{k}, \gamma_{k}\right)$ is the evolution of the stock price up to this moment, i.e. $s_{0}, s_{1}, \ldots, s_{k}$. In other words

$$
\beta_{k}=\beta_{k}\left(s_{0}, s_{1}, \ldots, s_{k}\right), \quad \gamma_{k}=\gamma_{k}\left(s_{0}, s_{1}, \ldots, s_{k}\right) .
$$

The player is called a writer (seller, investor).

A strategy is called self-financing if the changes of the portfolio content do not affect its value, i.e.

$$
\beta_{k} b_{k}+\gamma_{k} s_{k}=\beta_{k-1} b_{k}+\gamma_{k-1} s_{k}, \quad k=1, \ldots, n-1 .
$$

The final goal of the game is to meet the condition

$$
x_{n}=\beta_{n-1} b_{n}+\gamma_{n-1} s_{n} \geq f\left(s_{n}\right)
$$

where $f(s)$ is the so-called pay-off function of the simplest option of the European type having $n$ as maturity date. For more about the mathematical and substantial aspects of the option pricing theory see e.g. Shiryaev (1999).

Basic problems of the mathematical theory of options are the evaluation of the so-called rational option price and the corresponding strategy leading to (1.1). Recall that the rational option price is the minimal initial capital $x_{0}$ which allows the investor to meet contract terms under proper behavior.

Both the problems are easily solved in the so-called binary model, that is, in the case where $\xi_{k}$ takes only two values $d$ and $u, d<\varrho<u$. In this case (see e.g. Ch. VI in Shiryaev (1999))

$$
x_{0}=\varrho^{-n} \sum_{k=0}^{n} C_{n}^{k} p_{*}^{k}\left(1-p_{*}\right)^{n-k} f\left(s_{0} u^{k} d^{n-k}\right)
$$

where

$$
p_{*}=\frac{\varrho-d}{u-d} .
$$

It is worth emphasizing that (1.2) does not place any restrictions on the measure which governs the evolution of the stock price $\left(\xi_{1}, \ldots, \xi_{n}\right)$. Furthermore, there exists a unique self-financing strategy

$$
(\boldsymbol{\beta}, \boldsymbol{\gamma})=\left\{\left(\beta_{0}, \gamma_{0}\right),\left(\beta_{1}, \gamma_{1}\right), \ldots,\left(\beta_{n-1}, \gamma_{n-1}\right)\right\}
$$

leading to the equality

$$
x_{n}=\beta_{n-1} b_{n}+\gamma_{n-1} s_{n}=f\left(s_{n}\right) .
$$

The strategy is defined by the formulae

$$
\begin{aligned}
& \beta_{k}=\frac{u f_{k+1}\left(s_{k} d\right)-d f_{k+1}\left(s_{k} u\right)}{\varrho b_{k}(u-d)}, \\
& \gamma_{k}=\frac{f_{k+1}\left(s_{k} u\right)-f_{k+1}\left(s_{k} d\right)}{s_{k}(u-d)},
\end{aligned}
$$


where

$$
f_{k}(s)=\varrho^{-(n-k)} \sum_{j=0}^{n-k} C_{n-k}^{j} p_{*}^{j}\left(1-p_{*}\right)^{n-k-j} f\left(s u^{j} d^{n-k-j}\right) .
$$

The successive values of the portfolio are

$$
x_{k}=f_{k}\left(s_{k}\right), \quad k=0,1, \ldots, n-1 .
$$

If $\xi_{k}, k=1, \ldots, n$, take more than two values then it is impossible to guarantee the desired relation (1.3) with probability 1 . However, sometimes it is possible to guarantee (1.1). For example, if $\xi_{k} \in[d, u]$ and $f(s)$ is convex then the minimal initial capital is evaluated by the same formula (1.2).

This fact was first proven in Tessitore and Zabczyk (1996) (see also Zabczyk (1996) and Motoczyński and Stettner (1998)). The proof applies control theory methods. Later on in Shiryaev (1999) the rational price is derived as the solution of an extreme problem (see Theorem V.1c.1 ibidem). It seems that Shiryaev knew nothing about the works of his predecessors. At least in the rather rich list of references given in Shiryaev (1999) they are not mentioned.

\section{Define}

$$
\bar{x}_{k}=f_{k}\left(s_{k}\right), \quad k=0, \ldots, n-1,
$$

and let $\left(\beta_{k}, \gamma_{k}\right)$ be as defined in (1.4) and (1.5).

Possessing after the $(k-1)$ th step the capital $\bar{x}_{k-1}$ distributed in accordance with (1.4) and (1.5), at the next step $k$ the investor gains the capital

$$
x_{k}=\beta_{k-1} b_{k}+\gamma_{k-1} s_{k}=\frac{u-\xi_{k}}{u-d} f_{k}\left(s_{k-1} d\right)+\frac{\xi_{k}-d}{u-d} f_{k}\left(s_{k-1} u\right)
$$

(for more details see e.g. A. Nagaev and S. Nagaev (2000a,b)).

If $\xi_{k} \in[d, u], k=1, \ldots, n$, then

$$
\delta_{k}=x_{k}-\bar{x}_{k}=f_{k}\left(s_{k-1} d\right) \frac{u-\xi_{k}}{u-d}+f_{k}\left(s_{k-1} u\right) \frac{\xi_{k}-d}{u-d}-f_{k}\left(s_{k-1} \xi_{k}\right) \geq 0 .
$$

It is easily seen that $\delta_{k}=0$ if and only if $\xi_{k}=d$ or $\xi_{k}=u$. Otherwise $\delta_{k}>0$. Thus, if $\xi_{k}$ takes at least one value lying in $(d, u)$ then a profit arises. If the extreme values $d$ and $u$ belong to the support of the distribution of $\xi_{k}$ then $\bar{x}_{k-1}$ is the minimal capital which allows such a profit. This implies that the policy determined by (1.4) and (1.5) forms the so-called hedge or, in the terminology adopted in [1], upper hedge, while $\left(\bar{x}_{0}, \bar{x}_{1}, \ldots, \bar{x}_{n-1}\right)$ is the corresponding chain of hedging capitals.

The investor may take advantage of the profit so arising in various ways. The simplest one is to withdraw from the game the superfluous quota $\delta_{k}$ which till the maturity date acquires the value $\delta_{k} \varrho^{n-k}$. So, the self-financing condition is fulfilled only in the part which bans any capital inflows. 
Having withdrawn an unnecessary quota one should follow the "binary" optimal strategy determined by (1.4) and (1.5). As a result, till the maturity date the investor accumulates a riskless profit

$$
\Delta_{n}=\delta_{1} \varrho^{n-1}+\delta_{2} \varrho^{n-2}+\ldots+\delta_{n} .
$$

It is not easy to find the distribution of $\Delta_{n}$ even in the case of independent $\xi_{k}$. The question arises how to approximate it. To obtain such an approximation is a basic goal of the paper.

It is worth emphasizing that we consider the simplest case where the random variables $\xi_{k}, k=1, \ldots, n$, are i.i.d. In contrast to A. Nagaev and S. Nagaev (2002b) where the pay-off function is assumed to be smooth, here we focus on a typical example of a non-smooth pay-off function which is provided by the call option. Our basic goal is to show how the chaotic phenomena which arise here could be analyzed.

The paper is organized as follows. In Section 2 the basic results are formulated. The "local" profit in the case where the model converges to that of the geometrical Brownian motion is studied in Section 3. In Section 4 the limit of the expected value of the total riskless profit is established. The limit distribution of the total riskless profit is given in Section 5. Concluding remarks are gathered together in Section 6. Auxiliary facts are given in Appendices I and II.

2. Basic results. From now on we deal with the simplest case of the standard call option determined by the pay-off function

$$
f(s)=(s-K)_{+} \cdot
$$

Put in (1.9),

$$
\left\{\begin{array}{l}
u=u_{n}=\exp \left(\mu n^{-1}+x n^{-1 / 2}\right)=1+x n^{-1 / 2}+O\left(n^{-1}\right), \\
d=d_{n}=\exp \left(\mu n^{-1}-x n^{-1 / 2}\right)=1-x n^{-1 / 2}+O\left(n^{-1}\right), \\
\varrho=\varrho_{n}=\exp \left(\alpha n^{-1}\right)=1+\alpha n^{-1}+O\left(n^{-2}\right), \\
\xi_{k}=\xi_{k, n}=\exp \left(\mu n^{-1}+\eta_{k} n^{-1 / 2}\right)=1+\eta_{k} n^{-1 / 2}+\left(\mu+2 \theta x^{2}\right) n^{-1}, \\
s_{k, n}=s_{0} \xi_{1, n} \ldots \xi_{k, n},
\end{array}\right.
$$

where $\alpha>0$ and $\mu$ are constants, $|\theta| \leq 1$, while the random variables $\eta_{k}, k=1, \ldots, n$, are i.i.d. in $[-x, x]$, so that $\mathrm{E} \eta_{1}=0, \operatorname{Var} \eta_{1}=\sigma^{2}>0$. We also assume that the extreme points $\pm x$ belong to the support of the distribution of $\eta_{1}$.

Consider the stochastic process

$$
\begin{aligned}
x_{n}(t) & =\ln \left(s_{k, n} / s_{0}\right) \\
& =\frac{\eta_{1}+\ldots+\eta_{k}}{\sqrt{n}}+\mu k n^{-1}, \quad(k-1) n^{-1} \leq t<k n^{-1}, k=1, \ldots, n .
\end{aligned}
$$


It is well known that $x_{n}(t)$ weakly converges to $x(t)=\mu t+\sigma w(t)$ where $w(t)$ is the standard Wiener process (see e.g. Billingsley (1968), Ch. 3).

Define

$$
\begin{aligned}
\psi(t, v)= & 2(1-t)^{-1 / 2} \varphi\left((1-t)^{-1 / 2}\left(\frac{\ln K}{x}-\frac{v}{x}\right)\right. \\
& \left.+(1-t)^{1 / 2}\left(\frac{x}{2}-\frac{\alpha}{x}\right)\right) \\
I(t)= & \mathrm{E} \psi\left(t, x(t)+\ln s_{0}\right) \\
= & \frac{2 x}{\sqrt{t \sigma^{2}+x^{2}(1-t)}} \varphi\left(\frac{\ln \left(K / s_{0}\right)-\mu t-(1-t)\left(\alpha-x^{2} / 2\right)}{\sqrt{t \sigma^{2}+x^{2}(1-t)}}\right) .
\end{aligned}
$$

Here $\varphi(v)$ is the density of the standard normal law. Further, let

$$
a_{F}=\int_{0}^{1}\left(u \int_{2 x(u-1 / 2)}^{x}(x-y) d F(y)+(1-u) \int_{-x}^{2 x(u-1 / 2)}(y+x) d F(y)\right) d u
$$

where $F$ is the distribution function of $\eta_{1}$.

The following two theorems contain the basic results of the present paper.

Theorem 2.1. Let the distribution of $\eta_{1}$ be non-lattice, i.e.

$$
\left|\mathrm{E} e^{\imath t \eta_{1}}\right| \neq 1 \quad \text { for all } t \neq 0 .
$$

Then as $n \rightarrow \infty$,

$$
\mathrm{E} \Delta_{n}=K a_{F} \int_{0}^{1} I(t) d t+o(1)
$$

where $K$ is the strike price from (2.10).

Theorem 2.2. Under the conditions of Theorem 2.1,

$$
\Delta_{n} \stackrel{d}{\rightarrow} l(x(t))=K a_{F} \int_{0}^{1} \psi\left(t, x(t)+\ln s_{0}\right) d t
$$

where

$$
x(t)=\mu t+\sigma w(t),
$$

$w(t)$ is the standard Wiener process and $\psi(t, z)$ is as defined in (2.12).

3. "Local" profit of the investor. Let us convene to denote by $c$ any positive constant whose concrete value is of no importance. Under this convention we have e.g. $c+c=c, c^{2}=c$ etc. By $\theta$ we denote any variables taking values in $[-1,1]$. 
Define

$$
\begin{aligned}
p_{n} & =\frac{\varrho_{n}-d_{n}}{u_{n}-d_{n}}, & \lambda_{k, n} & =\frac{\xi_{k, n}-d_{n}}{u_{n}-d_{n}}, \\
a_{j, m} & =u_{n}^{j} d_{n}^{m-j}, & b_{j, m} & =C_{m}^{j} p_{n}^{j}\left(1-p_{n}\right)^{m-j} .
\end{aligned}
$$

Then the discounted "local" profit of the investor takes the form

$$
\begin{aligned}
\Delta_{k, n}= & \delta_{k, n} \varrho_{n}^{n-k} \\
= & \sum_{j=0}^{n-k} b_{j, n-k}\left(\lambda_{k, n} f\left(s_{k-1, n} u_{n} a_{j, n-k}\right)\right. \\
& \left.\quad+\left(1-\lambda_{k, n}\right) f\left(s_{k-1, n} d_{n} a_{j, n-k}\right)-f\left(s_{k-1, n} \xi_{k, n} a_{j, n-k}\right)\right) .
\end{aligned}
$$

For the time being we suppress the dependence of $\lambda, d, u, \xi_{k}$ and $s_{k}$ on $n$. Let $j$ be such that $s_{k-1} d a_{j, n-k}>K$. Then

$$
\begin{aligned}
\lambda_{k} f\left(s_{k-1} u a_{j, n-k}\right)+\left(1-\lambda_{k}\right) f\left(s_{k-1} d a_{j, n-k}\right)-f\left(s_{k-1} \xi_{k} a_{j, n-k}\right) & \\
& =s\left(\lambda_{k} u+\left(1-\lambda_{k}\right) d-\xi_{k}\right) a_{j, n-k}=0 .
\end{aligned}
$$

If $s_{k-1} u a_{j, n-k}<K$ then

$$
0=f\left(s_{k-1} u a_{j, n-k}\right) \geq f\left(s_{k-1} \xi_{k} a_{j, n-k}\right) \geq f\left(s_{k-1} d a_{j, n-k}\right) .
$$

It is worth reminding that $d \leq \xi_{k-1} \leq u$. Thus,

$$
\begin{aligned}
& \Delta_{k, n}=\delta_{k, n} \varrho_{n}^{n-k}=\sum_{r_{n-k}(u)<j \leq r_{n-k}(d)} b_{j, n-k}\left(\lambda_{k}\left(s_{k-1} u a_{j, n-k}-K\right)_{+}\right. \\
& \left.+\left(1-\lambda_{k}\right)\left(s_{k-1} d a_{j, n-k}-K\right)_{+}-\left(s_{k-1} \xi_{k} a_{j, n-k}-K\right)_{+}\right)
\end{aligned}
$$

where

$$
r_{m}(z)=r_{m}\left(z, s_{k-1}\right)=\frac{\ln \left(K /\left(s_{k-1} z d^{m}\right)\right)}{\ln (u / d)} .
$$

Further,

$$
\begin{aligned}
& \Delta_{k, n}=\sum_{r_{n-k}\left(\xi_{k}\right)<j \leq r_{n-k}(d)} b_{j, n-k}\left(\lambda_{k}\left(s_{k-1} u a_{j, n-k}-K\right)\right. \\
& \left.-\left(s_{k-1} \xi_{k} a_{j, n-k}-K\right)\right) \\
& +\lambda_{k} \sum_{r_{n-k}(u)<j \leq r_{n-k}\left(\xi_{k}\right)} b_{j, n-k}\left(s_{k-1} u a_{j, n-k}-K\right) \\
& =\Delta_{k, n}^{\prime}+\Delta_{k, n}^{\prime \prime} .
\end{aligned}
$$

It is easily verified that

$$
\begin{aligned}
& \Delta_{k, n}^{\prime}=\left(1-\lambda_{k}\right) K \sum_{r_{n-k}\left(\xi_{k}\right)<j \leq r_{n-k}(d)} b_{j, n-k}\left(1-(d / u)^{r_{n-k}(d)-j}\right), \\
& \Delta_{k, n}^{\prime \prime}=\lambda_{k} K \sum_{r_{n-k}(u)<j \leq r_{n-k}\left(\xi_{k}\right)} b_{j, n-k}\left((u / d)^{j+1-r_{n-k}(d)}-1\right) .
\end{aligned}
$$


In view of (7.42) and (7.44) we have uniformly in $k$, for $\delta n \leq k \leq(1-\delta) n$,

$$
\begin{gathered}
1-(d / u)^{r_{n-k}(d)-j}=2 x n^{-1 / 2}\left(r_{n-k}(d)-j+O\left(n^{-1}\right)\right), \\
(u / d)^{j+1-r_{n-k}(d)}-1=2 x n^{-1 / 2}\left(j+1-r_{n-k}(d)+O\left(n^{-1}\right)\right) .
\end{gathered}
$$

Taking into account (2.11) we conclude that

$$
\begin{aligned}
\Delta_{k, n}^{\prime}= & K\left(x-\eta_{k}+\left(\mu+2 \theta X^{2}\right) n^{-1 / 2}\right) n^{-1 / 2} \\
& \times \sum_{r_{n-k}\left(\xi_{k}\right)<j \leq r_{n-k}(d)} b_{j, n-k}\left(r_{n-k}(d)-j+O\left(n^{-1}\right)\right), \\
\Delta_{k, n}^{\prime \prime}= & K\left(\eta_{k}+x+\left(\mu+2 \theta X^{2}\right) n^{-1 / 2}\right) n^{-1 / 2} \\
& \times \sum_{r_{n-k}(u)<j \leq r_{n-k}\left(\xi_{k}\right)} b_{j, n-k}\left(j+1-r_{n-k}(d)+O\left(n^{-1}\right)\right) .
\end{aligned}
$$

Both the representations are valid uniformly in $k$ for $\delta n \leq k \leq(1-\delta) n$.

From (7.47) and the uniform version of the Moivre-Laplace local limit theorem we obtain, for $\delta n \leq k \leq(1-\delta) n$,

$$
b_{j, n-k}=n^{-1 / 2} \psi\left(k n^{-1}, \ln s_{k-1}\right)+o\left(n^{-1 / 2}\right) .
$$

It is worth emphasizing that (3.17) holds uniformly in $s_{k-1}$. Thus,

$$
\begin{aligned}
\Delta_{k, n}^{\prime}= & K\left(x-\eta_{k}\right) n^{-1} \psi\left(k n^{-1}, \ln s_{k-1}\right) \\
& \times \sum_{r_{n-k}\left(\xi_{k}\right)<j \leq r_{n-k}(d)}\left(r_{n-k}(d)-j\right)+O\left(n^{-3 / 2}\right), \\
\Delta_{k, n}^{\prime \prime}= & K\left(\eta_{k}+x\right) n^{-1} \psi\left(k n^{-1}, \ln s_{k-1}\right) \\
& \times \sum_{r_{n-k}(u)<j \leq r_{n-k}\left(\xi_{k}\right)}\left(j+1-r_{n-k}(d)\right)+O\left(n^{-3 / 2}\right) .
\end{aligned}
$$

Both the representations are valid uniformly in $k$ for $\delta n \leq k \leq(1-\delta) n$.

Let $\delta^{\prime} \leq\left\{r_{n-k}(d)\right\} \leq 1-\delta^{\prime}$ and suppose both $n$ and $n-k$ are sufficiently large. Then by (7.46) the interval $\left(r_{n-k}(u), r_{n-k}(d)\right]$ contains exactly one integer $j=\left[r_{n-k}(d)\right]$. So,

$$
\sum_{r_{n-k}\left(\xi_{k}\right)<j \leq r_{n-k}(d)}\left(r_{n-k}(d)-j\right)= \begin{cases}\left\{r_{n-k}(d)\right\} & \text { if } r_{n-k}\left(\xi_{k}\right)<\left[r_{n-k}(d)\right], \\ 0 & \text { otherwise. }\end{cases}
$$

Similarly,

$$
\sum_{r_{n-k}(u)<j \leq r_{n-k}\left(\xi_{k}\right)}\left(j+1-r_{n-k}(d)\right)= \begin{cases}0 & \text { if } r_{n-k}\left(\xi_{k}\right)<\left[r_{n-k}(d)\right], \\ 1-\left\{r_{n-k}(d)\right\} & \text { otherwise. }\end{cases}
$$

For $\delta^{\prime} \leq\left\{r_{n-k}(d)\right\} \leq 1-\delta^{\prime}$ we may combine (3.16) and the last estimates in the following way:

$$
\Delta_{k, n}=K n^{-1} \psi\left(k n^{-1}, \ln s_{k-1}\right) \sigma_{k, n}+O\left(n^{-3 / 2}\right)
$$


where

$$
\sigma_{k, n}= \begin{cases}\left(x-\eta_{k}\right)\left\{r_{n-k}(d)\right\} & \text { if } r_{n-k}\left(\xi_{k}\right)<\left[r_{n-k}(d)\right] \\ \left(\eta_{k}+x\right)\left(1-\left\{r_{n-k}(d)\right\}\right) & \text { otherwise. }\end{cases}
$$

Therefore,

$$
\Delta_{k, n}=K n^{-1} \psi\left(k n^{-1}, \ln s_{k-1}\right) \sigma_{k, n}+K^{\prime} \theta n^{-1} \mathbf{1}_{k, n}+c \theta n^{-3 / 2}
$$

where

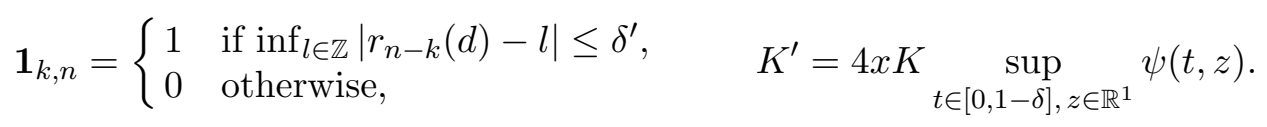

It is convenient to replace $\sigma_{k, n}$ by

$$
\sigma_{k, n}^{*}= \begin{cases}\left(x-\eta_{k}\right)\left\{r_{n-k}(d)\right\} & \text { if } \eta_{k}>2 x\left(\left\{r_{n-k}(d)\right\}-1 / 2\right), \\ \left(\eta_{k}+x\right)\left(1-\left\{r_{n-k}(d)\right\}\right) & \text { otherwise. }\end{cases}
$$

In order to justify the replacement note that in view of $(7.43), r_{n-k}(z)<$ $\left[r_{n-k}(d)\right]$ with $z=1+w n^{-1 / 2}$ implies

$$
-\frac{w}{2 x}+O\left(n^{-1 / 2} \ln n\right)<1 / 2-\left\{r_{n-k}(d)\right\}
$$

or equivalently,

$$
w>2 x\left(\left\{r_{n-k}(d)\right\}-1 / 2\right)+O\left(n^{-1 / 2} \ln n\right) .
$$

Obviously,

where

$$
\left|\sigma_{k, n}^{*}-\sigma_{k, n}\right| \leq 2 x \mathbf{1}_{k, n}^{\prime}
$$

$$
\mathbf{1}_{k, n}^{\prime}= \begin{cases}1 & \text { if }\left|\eta_{k}-2 x\left(\left\{r_{n-k}(d)\right\}-1 / 2\right)\right| \leq c n^{-1 / 2} \ln n \\ 0 & \text { otherwise }\end{cases}
$$

So, we arrive at the following final representation of the "local" profit:

(3.19) $\Delta_{k, n}=K n^{-1} \psi\left(k n^{-1}, \ln s_{k-1}\right) \sigma_{k, n}^{*}+K^{\prime} \theta n^{-1}\left(\mathbf{1}_{k, n}+\mathbf{1}_{k, n}^{\prime}\right)+c \theta n^{-3 / 2}$.

It remains to estimate the expectations of $\mathbf{1}_{k, n}$ and $\mathbf{1}_{k, n}^{\prime}$. By Lemma 7.1 for all sufficiently large $n$ and $k$,

$$
\mathrm{E} \mathbf{1}_{k, n} \leq 4 \delta^{\prime}
$$

Further,

$$
\begin{aligned}
& \mathrm{E}_{k, n}^{\prime}=\int_{-x}^{x} d F(v) \mathrm{P}\left(\left|v-2 x\left(\left\{r_{n-k}(d)\right\}-1 / 2\right)\right| \leq c n^{-1 / 2} \ln n\right) \\
& =\int_{-x}^{x} d F(v) \mathrm{P}\left(\frac{1}{2}+\frac{v}{2 x}-c n^{-1 / 2} \ln n \leq\left\{r_{n-k}(d)\right\} \leq \frac{1}{2}+\frac{v}{2 x}+c n^{-1 / 2} \ln n\right) .
\end{aligned}
$$

By (7.43),

$$
\left\{r_{n-k}(d)\right\}=\left\{\frac{\eta_{1}+\ldots+\eta_{k-1}}{2 x}+a_{k, n}\right\}
$$


Therefore, by Lemma 7.1, $\left\{r_{n-k}(d)\right\}$ is asymptotically uniformly distributed. This implies that for any $\delta^{\prime}$ and all sufficiently large $n$ and $k$,

$$
\mathrm{E} \mathbf{1}_{k, n}^{\prime} \leq 2 \delta^{\prime}
$$

4. Proof of Theorem 2.1. We represent the total profit $\Delta_{n}$ as

$$
\begin{aligned}
\Delta_{n} & =\sum_{1 \leq k<\delta n} \Delta_{k, n}+\sum_{\delta n \leq k \leq(1-\delta) n} \Delta_{k, n}+\sum_{(1-\delta) n \leq k \leq n} \Delta_{k, n} \\
& =\Delta_{n}^{\prime}+\Delta_{n}^{\prime \prime}+\Delta_{n}^{\prime \prime \prime}
\end{aligned}
$$

and estimate the expectations $\mathrm{E} \Delta_{n}^{\prime}, \mathrm{E} \Delta_{n}^{\prime \prime}$ and $\mathrm{E} \Delta_{n}^{\prime \prime \prime}$ one after another.

According to (3.19) we have

$$
\begin{aligned}
\mathrm{E} \Delta_{n}^{\prime \prime}= & K n^{-1} \sum_{\delta n \leq k \leq(1-\delta) n} \mathrm{E} \psi\left(k n^{-1}, \ln s_{k-1, n}\right) \sigma_{k, n}^{*} \\
& +K^{\prime} \theta n^{-1} \sum_{\delta n \leq k \leq(1-\delta) n} \mathrm{E}\left(\mathbf{1}_{k, n}+\mathbf{1}_{k, n}^{\prime}\right)+c \theta n^{-1 / 2} .
\end{aligned}
$$

In view of (3.20) and (3.21) for any $\delta^{\prime}>0$,

$$
\begin{aligned}
\mathrm{E} \Delta_{n}^{\prime \prime}= & K n^{-1} \sum_{\delta n \leq k \leq(1-\delta) n} \mathrm{E} \psi\left(k n^{-1}, \frac{\eta_{1}+\ldots+\eta_{k-1}}{\sqrt{n}}+\mu \frac{k-1}{n}+\ln s_{0}\right) \sigma_{k, n}^{*} \\
& +c \theta \delta^{\prime}+c \theta n^{-1 / 2}
\end{aligned}
$$

Consider

$$
\begin{array}{r}
A(u, y)=(x-y) u \chi(u, y)+(y+x)(1-u)(1-\chi(u, y)), \\
(u, y) \in[0,1] \times[-x, x],
\end{array}
$$

where

$$
\chi(u, y)= \begin{cases}1 & \text { if } 2 x(u-1 / 2)<y \leq x, 0 \leq u \leq 1 \\ 0 & \text { if }-x<y \leq 2 x(u-1 / 2), 0 \leq u \leq 1\end{cases}
$$

It is evident that $\chi(u, y)$ admits a monotone $\varepsilon$-approximation by means of $\chi_{+}(u, y)$ and $\chi_{-}(u, y)$ where

$$
\begin{aligned}
& \chi_{+}(u, y)= \begin{cases}\frac{-2 x u+y+x}{\varepsilon}+1 & \text { if } 2 x(u-1 / 2)-\varepsilon \leq y \leq 2 x(u-1 / 2), \\
0 & \text { if }-x \leq y \leq 2 x(u-1 / 2)-\varepsilon, 0 \leq u \leq 1, \\
1 & \text { if } 2 x(u-1 / 2) \leq y \leq x, 0 \leq u \leq 1,\end{cases} \\
& \chi_{-}(u, y)= \begin{cases}\frac{-2 x u+y+x}{\varepsilon} & \text { if } 2 x(u-1 / 2) \leq y \leq 2 x(u-1 / 2)+\varepsilon, \\
0 & \text { if }-x \leq y \leq 2 x(u-1 / 2), 0 \leq u \leq 1, \\
1 & \text { if } 2 x(u-1 / 2)+\varepsilon \leq y \leq x, 0 \leq u \leq 1 .\end{cases}
\end{aligned}
$$


Obviously, $\chi_{ \pm}(u, y)$ are continuous in $[0,1] \times[-x, x]$ and

$$
\chi_{-}(u, y) \leq \chi(u, y) \leq \chi_{+}(u, y) .
$$

Furthermore,

$$
0 \leq \int_{[0,1] \times[-x, x]}\left(\chi_{+}(u, y)-\chi_{-}(u, y)\right) d u d F(y) \leq \int_{U_{\varepsilon}} d u d F(y) \leq \varepsilon / x
$$

where

$$
U_{\varepsilon}=\{(u, y): u \in(0,1),|y|<x,|y-2 x(u-1 / 2)| \leq \varepsilon\} .
$$

Therefore

$$
\begin{aligned}
& \mathrm{E} \psi\left(k n^{-1}, \frac{\eta_{1}+\ldots+\eta_{k-1}}{\sqrt{n}}+\mu \frac{k-1}{n}+\ln s_{0}\right) A_{-}\left(\left\{r_{n-k}(d)\right\}, \eta_{k}\right) \\
& \quad \leq \mathrm{E} \psi\left(k n^{-1}, \frac{\eta_{1}+\ldots+\eta_{k-1}}{\sqrt{n}}+\mu \frac{k-1}{n}+\ln s_{0}\right) \sigma_{k, n}^{*} \\
& \quad=\mathrm{E} \psi\left(k n^{-1}, \frac{\eta_{1}+\ldots+\eta_{k-1}}{\sqrt{n}}+\mu \frac{k-1}{n}+\ln s_{0}\right) A\left(\left\{r_{n-k}(d)\right\}, \eta_{k}\right) \\
& \quad \leq \mathrm{E} \psi\left(k n^{-1}, \frac{\eta_{1}+\ldots+\eta_{k-1}}{\sqrt{n}}+\mu \frac{k-1}{n}+\ln s_{0}\right) A_{+}\left(\left\{r_{n-k}(d)\right\}, \eta_{k}\right)
\end{aligned}
$$

where

$$
A_{ \pm}(u, y)=(x-y) u \chi_{ \pm}(u, y)+(y+x)(1-u)\left(1-\chi_{ \pm}(u, y)\right) .
$$

Obviously, the family $\psi(t, z), \delta \leq t \leq 1-\delta$, is contained in the class $\mathcal{G}$ defined in Appendix II. So, we may apply Corollary 7.2 to obtain

$$
\begin{aligned}
& \mathrm{E} \psi\left(k n^{-1}, \frac{\eta_{1}+\ldots+\eta_{k-1}}{\sqrt{n}}+\mu \frac{k-1}{n}+\ln s_{0}\right) A_{ \pm}\left(\left\{r_{n-k}(d)\right\}, \eta_{k}\right) \\
& =\mathrm{E} \psi\left(k n^{-1}, \sigma \nu \sqrt{k n^{-1}}+\mu k n^{-1}+\ln s_{0}\right) \int_{[0,1] \times[-x, x]} A_{ \pm}(u, y) d u d F(y)+o(1)
\end{aligned}
$$

uniformly in $k$ for $\delta \leq k n^{-1} \leq 1-\delta$. Here $\nu$ has the standard (0,1)-normal distribution. In view of (4.24),

$$
\int_{[0,1] \times[-x, x]} A_{ \pm}(u, y) d u d F(y)=a_{F}+\theta \varepsilon
$$

where $a_{F}$ is as defined in (2.14). Since $\varepsilon$ is arbitrary we obtain

$$
\begin{aligned}
\mathrm{E} \psi\left(k n^{-1}, \sigma \nu \sqrt{k n^{-1}}\right. & \left.+\mu k n^{-1}+\ln s_{0}\right) \sigma_{k, n}^{*} \\
& =a_{F} \mathrm{E} \psi\left(k n^{-1}, \sigma \nu \sqrt{k n^{-1}}+\mu k n^{-1}+\ln s_{0}\right)+o(1)
\end{aligned}
$$


uniformly in $k$ for $\delta \leq k n^{-1} \leq 1-\delta$. Thus,

$$
\begin{aligned}
\mathrm{E} \Delta_{n}^{\prime \prime}= & K n^{-1} a_{F} \sum_{\delta n \leq k \leq(1-\delta) n} \mathrm{E} \psi\left(k n^{-1}, \sigma \nu \sqrt{k n^{-1}}+\mu k n^{-1}+\ln s_{0}\right) \\
& +c \theta \delta^{\prime}+o(1) .
\end{aligned}
$$

Obviously,

$$
I(t)=\mathrm{E} \psi\left(t, \sigma \nu \sqrt{t}+\mu t+\ln s_{0}\right)=\int \psi\left(t, \sigma v \sqrt{t}+\mu t+\ln s_{0}\right) \varphi(v) d v
$$

or after straightforward calculations,

$$
I(t)=\frac{2 x}{\sqrt{t \sigma^{2}+x^{2}(1-t)}} \varphi\left(\frac{\ln \left(K / s_{0}\right)-\mu t-(1-t)\left(\alpha-x^{2} / 2\right)}{\sqrt{t \sigma^{2}+x^{2}(1-t)}}\right),
$$

whence we deduce

$$
\mathrm{E} \Delta_{n}^{\prime \prime}=K a_{F} \int_{\delta}^{1-\delta} I(t) d t+c \theta \delta^{\prime}+o(1)
$$

Now we are going to estimate E $\Delta_{n}^{\prime \prime \prime}$. For the extreme "local" profit $\Delta_{n, n}$ we obtain

$$
\begin{aligned}
\Delta_{n, n}=\delta_{n, n}= & \left(s_{n-1, n} d_{n}-K\right)_{+} \frac{u_{n}-\xi_{n}}{u_{n}-d_{n}}+\left(s_{n-1, n} u_{n}-K\right)_{+} \frac{\xi_{n}-d_{n}}{u_{n}-d_{n}} \\
& -\left(s_{n-1, n} \xi_{n . n}-K\right)_{+}
\end{aligned}
$$

whence

$$
\Delta_{n, n}= \begin{cases}0 & \text { if } s_{n-1, n} u_{n} \leq K \text { or } s_{n-1, n} d_{n}>K \\ \theta\left(s_{n-1, n} u_{n}-K\right) & \text { if } K / u_{n}<s_{n-1, n} \leq K / d_{n}\end{cases}
$$

Therefore,

$$
\mathrm{E} \Delta_{n, n} \leq K\left(u_{n} / d_{n}-1\right) \leq c n^{-1 / 2} .
$$

For $m=n-k \geq 1$, in view of (3.16) and (7.45),

$$
\Delta_{n-m, n} \leq c \max _{j} b_{j, m}\left(\left(u_{n} / d_{n}\right)^{2}-1\right),
$$

or taking into account (7.48),

$$
\Delta_{n-m, n} \leq c m^{-1 / 2} n^{-1 / 2} .
$$

Thus, for all sufficiently large $n$,

$$
\mathrm{E} \Delta_{n}^{\prime \prime \prime} \leq c \delta^{1 / 2}
$$

Similarly,

$$
\mathrm{E} \Delta_{n}^{\prime} \leq c \delta
$$

Since $\delta$ and $\delta^{\prime}$ are arbitrary, in view of (4.22), (4.25), (4.27) and (4.28) the theorem follows. 
5. The limit distribution of the riskless profit. Consider the representation (4.22). Put

$$
\Delta_{n}^{*}=n^{-1} \sum_{\delta n \leq k \leq(1-\delta) n} \psi\left(k n^{-1}, \ln s_{k-1, n}\right) \sigma_{k, n}^{*} .
$$

Represent $\Delta_{n}^{*}$ as

(5.30) $\Delta_{n}^{*}=a_{F} n^{-1} \sum_{\delta n \leq k \leq(1-\delta) n} \psi\left(k n^{-1}, \ln s_{k-1, n}\right)\left(\sigma_{k, n}^{*}-a_{F}\right)=a_{F} l_{1, n}+l_{2, n}$.

By (2.12) we have

$$
\sup _{z} \psi(t, z) \leq(2 \pi(1-t))^{-1 / 2}
$$

In view of (4.27) and (4.28),

$$
l_{1, n}=n^{-1} \sum_{1 \leq k \leq n} \psi\left(k n^{-1}, \ln s_{k-1, n}\right)+\theta c \delta^{1 / 2}=l_{n}^{*}+\theta c \delta^{1 / 2} .
$$

We are going to prove that

$$
\mathrm{E} l_{2, n}^{2} \leq c \delta
$$

In view of (3.20), (3.21), (4.27), (4.28) and (5.33),

$$
\Delta_{n}=K a_{F} l_{n}^{*}+\omega\left(n, \delta, \delta^{\prime}\right)
$$

where

$$
\mathrm{E}\left|\omega\left(n, \delta, \delta^{\prime}\right)\right| \leq c\left(\delta^{1 / 2}+\delta^{\prime}\right) .
$$

Therefore, for any $\varepsilon>0$ and $\delta^{\prime \prime}>0$,

$$
\mathrm{P}\left(\left|\Delta_{n}-K a_{F} l_{n}^{*}\right| \geq \varepsilon\right) \leq \frac{c\left(\delta^{1 / 2}+\delta^{\prime}\right)}{\varepsilon} \leq \delta^{\prime \prime}
$$

provided $\delta$ and $\delta^{\prime}$ are sufficiently small. This implies that the limit distributions of $\Delta_{n}$ and $K a_{F} l_{n}^{*}$ coincide.

First, we tackle $l_{n}^{*}$. Consider the stochastic process

$$
\begin{aligned}
x_{n}(t) & =\ln \left(s_{k, n} / s_{0}\right) \\
& =\frac{\eta_{1}+\ldots+\eta_{k}}{\sqrt{n}}+\mu k n^{-1}, \quad(k-1) n^{-1} \leq t<k n^{-1}, k=1, \ldots, n .
\end{aligned}
$$

Obviously,

$$
l_{n}^{*}=\int_{0}^{1} \psi\left(t, x_{n}(t)+\ln s_{0}\right) d t .
$$

From (5.31) it follows that $l_{n}^{*}$ is a continuous functional on $D[0,1]$. Since $x_{n}(t)$ weakly converges to $x(t)=\mu t+\sigma w(t)$ we conclude that

$$
l_{n}^{*} \stackrel{d}{\rightarrow} \int_{0}^{1} \psi\left(t, x(t)+\ln s_{0}\right) d t .
$$


It remains to establish (5.33). It is easily seen that

$$
\mathrm{E} l_{2, n}^{2}=n^{-2} \sum_{\delta \leq k n^{-1} \leq 1-\delta} \mathrm{E} \psi^{2}\left(k n^{-1}, \ln s_{k-1, n}\right)\left(\sigma_{k, n}^{*}-a_{F}\right)^{2}
$$

$+2 n^{-2} \sum_{\delta n \leq l<k \leq n(1-\delta)} \mathrm{E} \psi\left(\ln { }^{-1}, \ln s_{l-1, n}\right)\left(\sigma_{l, n}^{*}-a_{F}\right) \psi\left(k n^{-1}, \ln s_{k-1, n}\right)\left(\sigma_{k, n}^{*}-a_{F}\right)$ $=\Sigma_{1}+2 \Sigma_{2}$

Obviously,

$$
\Sigma_{1}=O\left(n^{-1}\right)
$$

Split $\Sigma_{2}$ in the following way:

(5.38) $\Sigma_{2}=n^{-2}$

$$
\sum_{\delta n \leq l<k \leq n(1-\delta), k-l<\delta n}+n^{-2} \sum_{\delta n \leq l<k \leq n(1-\delta), k-l \geq \delta n}=\Sigma_{21}+\Sigma_{22} .
$$

Obviously,

$$
\Sigma_{21} \leq c \delta
$$

So, it remains to estimate $\Sigma_{22}$. Utilizing (3.18) and (4.23) we get

$$
\begin{aligned}
M_{l, k, n}= & \mathrm{E} \psi\left(l n^{-1}, \ln s_{l-1, n}\right)\left(\sigma_{l, n}^{*}-a_{F}\right) \psi\left(k n^{-1}, \ln s_{k-1, n}\right)\left(\sigma_{k, n}^{*}-a_{F}\right) \\
= & \mathrm{E} \psi\left(l n^{-1}, \ln s_{l-1, n}\right)\left(A\left(\left\{r_{n-l}\right\}, \eta_{l}\right)-a_{F}\right) \\
& \times \psi\left(k n^{-1}, \ln s_{k-1, n}\right)\left(A\left(\left\{r_{n-k}(d)\right\}, \eta_{k}\right)-a_{F}\right) .
\end{aligned}
$$

Define

$$
F_{m, n}(z)=\mathrm{P}\left(n^{-1 / 2}\left(\eta_{1}+\ldots+\eta_{m}\right)+\ln s_{0}<z\right) .
$$

Then we may represent $M_{l, k, n}$ as

$$
\begin{aligned}
M_{l, k, n}= & \int_{\mathbb{R}^{1} \times[-x, x]} \psi\left(l n^{-1}, z^{\prime}+(l-1) n^{-1} \mu\right) \\
& \times\left(A\left(\left\{r_{n-l}(d)\right\}, y^{\prime}\right)-a_{F}\right) d F_{l-1, n}\left(z^{\prime}\right) d F\left(y^{\prime}\right) \\
& \times \int_{\mathbb{R}^{1} \times[-x, x]} \psi\left(k n^{-1}, z^{\prime}+z+n^{-1 / 2} y+(k-1) n^{-1} \mu\right) \\
& \times\left(A\left(\left\{r_{n-k}(d)\right\}, y\right)-a_{F}\right) d F_{k-l-1, n}(z) d F(y) .
\end{aligned}
$$

Note that given $n^{-1 / 2}\left(\eta_{1}+\ldots+\eta_{l-1}\right)=z^{\prime}, \eta_{l}=y^{\prime}$ we have

$$
\begin{aligned}
r_{n-k}(d) & =a_{k, n}-\frac{\eta_{1}+\ldots+\eta_{k-1}}{2 x}=a_{k, n}^{\prime}-\frac{\eta_{l+1}+\ldots+\eta_{k-1}}{2 x} \\
& \stackrel{d}{=} a_{k, n}^{\prime}-\frac{\eta_{1}+\ldots+\eta_{k-l-1}}{2 x} .
\end{aligned}
$$

Further, note that for $\delta \leq k n^{-1} \leq 1-\delta$ the functions $\psi\left(k n^{-1}, z^{\prime}+z\right),\left|z^{\prime}\right|<Z$, belong to the class $\mathcal{G}$ for any $Z>0$. Since $k-l \geq \delta n$ and $A(u, y)$ admits a monotone $\varepsilon$-approximation we may apply Corollary 7.2. Thus 


$$
\begin{array}{r}
\lim _{n \rightarrow \infty} \sup _{\delta n \leq l<k \leq n(1-\delta), k-l \geq \delta n} \sup _{\left|z^{\prime}\right|<Z} \mid \int_{\mathbb{R}^{1} \times[-x, x]} \psi\left(k n^{-1}, z^{\prime}+z+n^{-1 / 2} y+(k-1) n^{-1} \mu\right) \\
\times\left(A\left(\left\{r_{n-k}(d)\right\}, y\right)-a_{F}\right) d F_{k-l-1, n}(z) d F(y) \mid=0 .
\end{array}
$$

This implies that

$$
\Sigma_{22} \leq \sup _{\delta n \leq l<k \leq n(1-\delta), k-l \geq \delta n}\left|M_{l, k, n}\right|=o(1) .
$$

Combining (5.36)-(5.40) yields (5.33), which completes the proof of Theorem 2 .

6. Concluding remarks. - It is worth explaining why the total riskless profit does not vanish asymptotically. Since the pay-off function is piecewise linear the "local" profit $\Delta_{k, n}$ is determined by the summands in (3.15) whose labels lie between $r_{n-k}(d)$ and $r_{n-k}(u)$. By (7.44), typically, there is only one such label. The main point in our argument is the representation (3.19). Here the chaotic multiplier $\sigma_{k, n}^{*}$ highlights the role played by the support of the distribution of $\eta_{1}$. If the support contains only two points $\pm x$ then in view of (3.18) this term vanishes. But if the distribution of $\eta_{1}$ is supported by at least one more point then $\mathrm{P}\left(\sigma_{k, n}^{*}>0\right)>0$, and therefore, the order of the "local" profit is $O_{p}(1 / n)$. This explains why there exists a non-degenerate limit distribution for the total profit.

- It should be emphasized that the methods used in the paper can be applied to much more general schemes. In particular, the assumption that the stock price jumps are identically distributed is not necessary at all. Moreover, the independence can be replaced by a mixing condition. The authors intend to discuss this point elsewhere.

- Note also that the asymptotic analysis of the hedge and of the corresponding capitals is implemented by the same scheme. Substituting into (1.4) and (1.5) the values of the parameters from (2.11) we obtain the components of the optimal strategy in the form

$$
\begin{aligned}
\beta_{k, n} & =\frac{u_{n} \sum_{j=0}^{n-k} b_{j, n-k}\left(s_{k} d_{n} a_{j, n-k}-K\right)_{+}-d_{n} \sum_{j=0}^{n-k} b_{j, n-k}\left(s_{k} u_{n} a_{j, n-k}-K\right)_{+}}{\varrho_{n} b_{k, n}\left(u_{n}-d_{n}\right)}, \\
\gamma_{k, n} & =\frac{\sum_{j=0}^{n-k} b_{j, n-k}\left(\left(s_{k} u_{n} a_{j, n-k}-K\right)_{+}-\left(s_{k} d_{n} a_{j, n-k}-K\right)_{+}\right)}{s_{k}\left(u_{n}-d_{n}\right)} .
\end{aligned}
$$

Similarly, from (1.7) and (2.11) the hedging capitals are

$$
\bar{x}_{k, n}=\varrho^{-(n-k)} \sum_{j=0}^{n-k} b_{j, n-k}\left(s_{k, n} a_{j, n-k}-K\right)_{+}, \quad k=0, \ldots, n-1 .
$$


It makes no problem to verify that the vector-process

$$
\left(\beta_{n}(t), \gamma_{n}(t)\right)=\left(\beta_{k-1, n}, \gamma_{k-1, n}\right), \quad(k-1) n^{-1} \leq t<k n^{-1}, k=1, \ldots, n,
$$

weakly converges to $(\beta(t), \gamma(t)), 0 \leq t \leq 1$, where

$$
\beta(t)=\Phi\left(y_{+}\right), \quad \gamma(t)=-K e^{-\alpha} \Phi\left(y_{-}\right)
$$

with

$$
\begin{aligned}
& y_{+}=\frac{\ln (s(t) / K)+(1-t)\left(\alpha+x^{2} / 2\right)}{x \sqrt{1-t}}, \\
& y_{-}=\frac{\ln (s(t) / K)+(1-t)\left(\alpha-x^{2} / 2\right)}{x \sqrt{1-t}} .
\end{aligned}
$$

It is worth emphasizing that this convergence takes place under the only condition that $s_{n}(t) \stackrel{w}{\Rightarrow} s(t)$. It is of interest to compare the limit strategy with the classical formulae established for the geometrical Brownian motion

$$
s(t)=s_{0} \exp \left(\left(r-\sigma^{2}\right) t+\sigma w(t)\right), \quad 0 \leq t \leq 1
$$

(see e.g. Shiryaev (1999, (8.1b.21), (8.1b.23) and (8.1b.24))). Those formulae could arise here provided in (6.41) $x=\sigma$. Since $\sigma^{2}=$ Var $\eta_{1} \leq x$ this implies that the discrete time model must be binary. However in that case no riskless profit exists.

Assume that $\eta_{1}$ takes at least three values. Putting $\mu=\alpha-\sigma^{2} / 2$ results in the limit process being the geometrical Brownian motion. Obviously, the hedge-process $\bar{x}_{n}(t)$ weakly converges to

$$
\bar{x}(t)=\beta(t) e^{-\alpha t}+\gamma(t) s(t)=s(t) \Phi\left(y_{+}\right)-K e^{-\alpha(1-t)} \Phi\left(y_{-}\right) .
$$

In order to stress the dependence of $\bar{x}(t)$ on $x$ write

$$
\bar{x}(t)=R_{t}(x)=s(t) \Phi\left(b x^{-1}+a x\right)-K e^{-\alpha(1-t)} \Phi\left(b x^{-1}-a x\right)
$$

with

$$
a=(1 / 2) \sqrt{1-t}, \quad b=\frac{\ln (s(t) / K)+(1-t) \alpha}{\sqrt{1-t}} .
$$

After a simple algebra we obtain

$$
R_{t}^{\prime}(x)=2 a s(t) \phi\left(b x^{-1}+a x\right)>0 .
$$

Thus $\bar{x}(t)$ increases in $x$ for each $t \in[0,1]$ and $s(t)$. Since $x>\sigma$ this implies that (see Shiryaev $(1999,(8.1 b .21)))$

$$
\bar{x}(t)>s(t) \Phi\left(y_{+}^{\prime}\right)-K e^{-\alpha(1-t)} \Phi\left(y_{-}^{\prime}\right)
$$

where

$$
y_{+}=\frac{\ln (s(t) / K)+(1-t)\left(\alpha+\sigma^{2} / 2\right)}{\sigma \sqrt{1-t}},
$$

while 


$$
y_{-}=\frac{\ln (s(t) / K)+(1-t)\left(\alpha-\sigma^{2} / 2\right)}{\sigma \sqrt{1-t}} .
$$

In other words, the basic characteristics of the game do not converge to their counterparts for the continuous time model. Loosely speaking the optimal behaviour within the framework of the dicrete time model "converges" to a strategy which is far from being optimal for the limit model.

- Let us put in (2.11)

$$
\xi_{k}=\xi_{k, n}=\exp \left\{g\left((k+1) n^{-1}\right)-g\left(k n^{-1}\right)+\eta_{k} n^{-1 / 2}\right\}
$$

where $g(u)$ is continuous on $[0,1]$. Then the process

$$
s_{n}(t)=s_{k-1, n}, \quad(k-1) n^{-1} \leq t<k n^{-1}, k=1, \ldots, n,
$$

weakly converges to

$$
s_{g}(t)=s_{0} \exp \left(\int_{0}^{t} g(u) d u+\sigma w(t)\right), \quad t \in[0,1] .
$$

It is easily seen that such a replacement requires no alteration in the proof. As to the statements of Theorems 2.1 and 2.2 one should replace in (2.13) $\mu t$ by $g(t)$.

- It is evident that instead of the call option we could deal with the put option.

\section{Appendices}

Appendix I. Properties of $r_{m}(z)$. Assume that

$$
d=1-n^{-1 / 2} x, \quad u=1+n^{-1 / 2} x, \quad 0<x^{\prime} \leq x \leq x^{\prime \prime}<\infty .
$$

Set

$$
z=1+n^{-1 / 2} w, \quad-x \leq w \leq x .
$$

Consider

$$
r_{m}(z)=r_{m}\left(z, s_{k-1, n}\right)=\frac{\ln \left(K /\left(s_{k-1, n} z d^{m}\right)\right)}{\ln (u / d)} .
$$

It is easily seen that

$$
\ln (u / d)=2 x n^{-1 / 2}\left(1+\frac{x^{2}}{3 n}+O\left(n^{-3 / 2}\right)\right) .
$$

Therefore,

$$
\frac{\ln z}{\ln (u / d)}=\frac{w}{2 x}+O\left(n^{-1 / 2}\right), \quad \frac{\ln d}{\ln (u / d)}=-\frac{1}{2}+O\left(n^{-1 / 2}\right),
$$

while for $|\ln s|=O(\ln n)$,

$$
\frac{\ln (K / s)}{\ln (u / d)}=\ln (K / s) \frac{n^{1 / 2}}{2 x}+O\left(n^{-1 / 2} \ln n\right) .
$$


From the above relations it follows that for $m \leq n$,

$$
r_{m}(z)=\frac{m}{2}+n^{1 / 2}\left(\frac{\ln K}{2 x}-\frac{\ln s}{2 x}+\frac{m}{n} \cdot \frac{x}{4}\right)-\frac{w}{2 x}+O\left(n^{-1 / 2} \ln n\right) .
$$

In particular,

$$
r_{m}(d)-r_{m}(u)=1+O\left(n^{-1 / 2} \ln n\right) .
$$

Moreover, for all sufficiently large $n$,

$$
\#\left\{j: j \geq 0, r_{m}(u)<j \leq r_{m}(d)\right\} \leq 2 .
$$

But if $m \geq \delta n, \delta^{\prime} \leq\left\{r_{m}(d)\right\} \leq 1-\delta^{\prime}, \delta, \delta^{\prime}>0$, and $n$ is sufficiently large then

$$
\#\left\{j: j \geq 0, r_{m}(u)<j \leq r_{m}(d)\right\}=1 .
$$

Since

$$
p_{*}=\frac{\varrho-d}{u-d}=\frac{1}{2}+\frac{\alpha}{2 x} n^{-1 / 2}+O\left(n^{-1}\right)
$$

we have

$$
r_{m}(d)-m p_{*}=n^{1 / 2}\left(\frac{\ln K}{2 x}-\frac{\ln s}{2 x}+\frac{m}{n}\left(\frac{x}{4}-\frac{\alpha}{2 x}\right)\right)+O(1),
$$

and therefore,

$$
\begin{aligned}
& \frac{r_{m}(d)-m p_{*}}{\sqrt{m p_{*}\left(1-p_{*}\right)}} \\
& \quad=(m / n)^{-1 / 2}\left(\frac{\ln K}{x}-\frac{\ln s}{x}\right)+(m / n)^{1 / 2}\left(\frac{x}{2}-\frac{\alpha}{x}\right)+O\left(m^{-1 / 2}\right) .
\end{aligned}
$$

Appendix II. Local limit theorems. Let $\eta, \eta_{1}, \eta_{2}, \ldots$ be i.i.d. random variables such that

$$
\mathrm{E} \eta=0, \quad \operatorname{Var} \eta=\sigma^{2}<\infty
$$

Consider

$$
\zeta_{n}=\eta_{1}+\ldots+\eta_{n} .
$$

If the distribution of $\eta$ is non-lattice then for any fixed $y^{\prime}, y^{\prime \prime}$ with $0<y^{\prime}<$ $y^{\prime \prime}<\infty$,

$$
\sup _{x, y^{\prime} \leq y \leq y^{\prime \prime}}\left|\mathrm{P}\left(x \leq \zeta_{n}<x+y\right)-\frac{y}{\sigma \sqrt{n}} \varphi\left(\frac{x}{\sigma \sqrt{n}}\right)\right|=o\left(n^{-1 / 2}\right) .
$$

This is a slightly generalized version of the Shepp local limit theorem (see e.g. A. Nagaev (1973)).

Consider the sequence of measures

$$
Q_{n}(A)=\sigma \sqrt{2 \pi n} \mathrm{P}\left(\zeta_{n} \in A\right) .
$$


The statement (7.48) implies that $Q_{n}$ weakly converges to the Lebesgue measure, that is, for any continuous compactly supported function $g(u)$,

$$
\int g(u) Q_{n}(d u) \rightarrow \int g(u) Q(d u)
$$

Let $\mathcal{G}$ be the class of equicontinuous functions defined on $(-\infty, \infty)$ such that

$$
\lim _{t \rightarrow \infty} \sup _{g \in \mathcal{G}} \int_{|u|>t}|g(u)| d u=0 .
$$

It is easily seen that (7.49) holds uniformly in $G \in \mathcal{G}$. More precisely,

$$
\lim _{n \rightarrow \infty} \sup _{g \in \mathcal{G}}\left|\int g(u) Q_{n}(d u)-\int g(u) Q(d u)\right|=0 .
$$

Consider the family of the random variables $\tau_{n}(a)=\left\{\lambda \zeta_{n}+a\right\}, a \in \mathbb{R}_{1}$, where $\lambda \neq 1$ is constant. It is worth comparing the following statement with the basic result in S. V. Nagaev and Mukhin (1966).

LEMMA 7.1. If the distribution of $\eta$ is non-lattice then for any fixed $u^{\prime}, u^{\prime \prime}$ with $0<u^{\prime}<u^{\prime \prime}<1$ and $z^{\prime}, z^{\prime \prime}$ with $-\infty<z^{\prime}<z^{\prime \prime}<\infty$, $\sup _{a}\left|\mathrm{P}\left(u^{\prime} \leq \tau_{n}(a)<u^{\prime \prime}, z^{\prime} \leq n^{-1 / 2} \zeta_{n}<z^{\prime \prime}\right)-\left(u^{\prime \prime}-u^{\prime}\right)\left(\Phi\left(z^{\prime \prime} / \sigma\right)-\Phi\left(z^{\prime} / \sigma\right)\right)\right|$
$=o(1)$ as $n \rightarrow \infty$.

Proof. Let $k=k(a)=[a], \theta=\theta(a)=\{a\}$ and $\lambda>0$. It is easily seen that

$$
\begin{aligned}
P_{n}= & \mathrm{P}\left(u^{\prime} \leq \tau_{n}(a)<u^{\prime \prime}, z^{\prime} \leq n^{-1 / 2} \zeta_{n}<z^{\prime \prime}\right) \\
= & \sum_{k} \mathrm{P}\left(k+u^{\prime} \leq \lambda \zeta_{n}+a<k+u^{\prime \prime}, z^{\prime} n^{1 / 2} \leq \zeta_{n}<z^{\prime \prime} n^{1 / 2}\right) \\
= & \sum_{k^{\prime} \leq k \leq k^{\prime \prime}} \mathrm{P}\left(\frac{k+u^{\prime}-\theta}{\lambda} \leq \zeta_{n}<\frac{k+u^{\prime \prime}-\theta}{\lambda}\right) \\
& +\mathrm{P}\left(\frac{k^{\prime \prime}+u^{\prime \prime}-\theta}{\lambda} \leq \zeta_{n}<z^{\prime \prime} n^{1 / 2}\right)+\mathrm{P}\left(z^{\prime} n^{1 / 2} \leq \zeta_{n}<\frac{k^{\prime}+u^{\prime}-\theta}{\lambda}\right)
\end{aligned}
$$

where

$$
k^{\prime}=\min \left\{k: \frac{k+u^{\prime}-\theta}{\lambda} \geq z^{\prime} n^{1 / 2}\right\}, \quad k^{\prime \prime}=\max \left\{k: \frac{k+u^{\prime \prime}-\theta}{\lambda} \leq z^{\prime \prime} n^{1 / 2}\right\} .
$$

According to (7.48),

$$
P_{n}=\frac{u^{\prime \prime}-u^{\prime}}{\lambda \sigma \sqrt{n}} \sum_{k^{\prime} \leq k \leq k^{\prime \prime}} \varphi\left(\frac{k}{\lambda \sigma \sqrt{n}}\right)+O\left(n^{-1 / 2}\right) .
$$

It remains to recall that

$$
k^{\prime}=z^{\prime} \lambda \sqrt{n}(1+o(1)), \quad k^{\prime \prime}=z^{\prime \prime} \lambda \sqrt{n}(1+o(1)) .
$$

The lemma is proved. 
Lemma 7.1 has the following evident corollary (cf. (7.50)).

Corollary 7.2. Let $\chi(u, y)$ be a bounded continuous function defined on $[0,1] \times \mathbb{R}^{1}$. Under the conditions of Lemma 7.1 ,

$$
\begin{aligned}
& \lim _{n \rightarrow \infty} \sup _{g \in \mathcal{G}} \sup _{a} \mid \mathrm{E} g\left(n^{-1 / 2} \zeta_{n}\right) \chi\left(\left\{\lambda \zeta_{n}+a\right\}, \eta_{n}\right) \\
&-\int g(\sigma z) \varphi(z) d z \int_{[0,1] \times \mathbb{R}^{1}} \chi(u, y) d u d F(y) \mid=0
\end{aligned}
$$

where $F$ is the distribution function of $\eta$.

Acknowledgments. The authors are grateful to the referee whose numerous comments and suggestions have helped to improve the presentation.

\section{References}

P. Billingsley (1968), Convergence of Probability Measures, Wiley, New York.

M. Motoczyński and Ł. Stettner (1998), On option pricing in the multinomial Cox-RossRubinstein model, Appl. Math. (Warsaw) 25, 55-72.

A. V. Nagaev (1973), Remarks on multidimensional local theorems, Mat. Zametki 14, 559-563 (in Russian).

A. V. Nagaev and S. A. Nagaev (2002a), Two remarks on a discrete model of financial market, Èkonom. Mat. Metod. 38, no. 3, 129-133 (in Russian).

A. V. Nagaev and S. A. Nagaev (2002b), On the riskless profit of investor under smooth pay-off function, J. Math. Sci., to appear.

S. V. Nagaev and A. B. Mukhin (1966), A case of convergence to the uniform distribution on an interval, in: Limit Theorems and Statistical Inference, Fan, Tashkent, 113-117 (in Russian).

V. V. Petrov (1975), Sums of Independent Variables, Springer, Berlin.

A. N. Shiryaev (1999), Essentials of Stochastic Finance, World Sci., Singapore.

A. N. Shiryaev, Yu. M. Kabanov, O. D. Kramkov and A. V. Mel'nikov (1994), Towards a theory of pricing options of European and American types. II, Continuous time. Theory Probab. Appl. 39, 61-102.

G. Tessitore and J. Zabczyk (1996), Pricing options for multinomial models, Bull. Polish Acad. Sci. 44, 363-380.

J. Zabczyk (1996), Pricing options by dynamic programming, in: Stochastic Processes and Related Topics, H. J. Engelbert et al. (eds.), Stochastic Monogr. 10, Gordon and Breach, 55-72.

Faculty of Mathematics and Computer Science

Nicolaus Copernicus University

12/18 Chopin St.

87-100 Toruń, Poland

E-mail: nagaev@mat.uni.torun.pl
Institute of Advanced Studies 56 Stumpergasse 1060 Wien, Austria E-mail: sergej@ihs.ac.at

Received on 27.6.2002; 